\title{
Impact of Model for End-Stage Liver Disease allocation system on outcomes of deceased donor liver transplantation: A single-center experience
}

\author{
Han Sang Park, Jeong-Moo Lee, Kwangpyo Hong, Eui Soo Han, Suk Kyun Hong, \\ YoungRok Choi, Nam-Joon Yi, Kwang-Woong Lee, Kyung-Suk Suh \\ Department of Surgery, Seoul National University College of Medicine, Seoul, Korea
}

\begin{abstract}
Backgrounds/Aims: From June of 2016, the Model for End-Stage Liver Disease (MELD)-based allocation system replaced the ChildTurcotte-Pugh (CTP) score-based system for organ allocation liver in Korea. The aim of this study was to analyze changes in outcomes and arising issues before and after the implementation of the MELD system.

Methods: From June 2014 to June 2018, 129 patients were selected from recipients who underwent deceased donor liver transplantation (DDLT) in Seoul National University Hospital. Pediatric cases were excluded. According to the allocation system, patients were divided into two groups (52 in the MELD group and 77 in the CTP group).

Results: MELD scores of the two groups differed significantly $(37.8 \pm 2.0$ in the MELD group vs. $31.0 \pm 8.2$ in the CTP group; $p=$ 0.001). The etiology of patients was changed for liver transplantation. The proportion of alcoholic liver cirrhosis increased in the era of the MELD allocation system. However, proportions of hepatitis B related liver cirrhosis and hepatocellular carcinoma were decreased. Six-month mortality rate of the MELD group was $25.0 \%$, which was higher than that (11.7\%) of the CTP group $(p=0.022)$. The 90-day complication rate was significantly higher in the MELD group than in the CTP group (11.5\% vs. $2.6 \% ; p=0.040)$.

Conclusions: When the MELD allocation system was used to distribute livers to severely ill patients, it resulted in poorer outcomes after surgery and higher proportion of alcoholic cirrhosis. Thus, it is necessary to adjust the MELD allocation system so that outcomes after DDLT could be improved.
\end{abstract}

Key Words: Organ allocation; Liver transplantation; Model for end-stage liver disease

\section{INTRODUCTION}

Organ shortage is one of the most significant limitations of deceased donor liver transplantation. The current "sickest first" principle of deceased donor liver donor distribution is to provide liver to the most severely ill patient. Since the es-

Received: February 22, 2021, Revised: March 18, 2021,

Accepted: March 30, 2021

Corresponding author: Jeong-Moo Lee

Department of Surgery, Seoul National University College of Medicine, 101

Daehak-ro, Jongno-gu, Seoul 03080, Korea

Tel: +82-2-2072-2817, Fax: +82-2-766-3975, E-mail: Iulu5050@naver.com

ORCID: https://orcid.org/0000-0001-7806-8759

Copyright (C) The Korean Association of Hepato-Biliary-Pancreatic Surgery tablishment of Korean Network for Organ Sharing (KONOS) in 2000, organ allocation has controlled the national system. Child-Turcotte-Pugh (CTP) score-based long-term distribution system has been implemented in Korea for the past 16 years, along with the United States' allocation system [1,2]. However, the demand for the reorganization of the system is increased due to a concern that the CTP score might be too wide to reflect a patient's severity. A subjective judgment of a personal physician might intervene in judging the extent of ascites and consciousness status. The Model for End-Stage Liver Disease (MELD) score is a tool developed to predict the survival of patients with cirrhosis after three months. It shows a significant meaning in predicting the survival time after a liver transplantatio [3-6]. The use of the MELD score as an indicator of organ distribution for liver transplantation has emerged. The United Network for Organ Sharing (UNOS) began to allocate organs based on the MELD system in 2002 [7,8]. This system was in- 
troduced in Korea in June 2016. It is used for organ allocation for deceased donor liver transplantation $[2,9]$.

However, even in the United States where the MELD system was introduced, several problems have arisen. After the system was applied, there was an increase in patient's mortality related to the high severity of recipients and the low graft quality related to a long transport time. For this reason, in the United States, they continue to adjust the allocation system for improving outcomes of deceased donor liver transplantation by continuing to provide feedback through supplementary measures and analysis of results of people who have undergone a liver transplant $[7,10]$.

In Korea, after introducing the MELD system, several results have been reported. According to recent reports, although the waiting list mortality is decreased, postoperative mortality is increased because the risk of surgery for recipients is also increased [2,9]. There has not been any feedback on this or any mention of problems when the MELD system is applied in Korea. Also, since there are few organ donors, patients with relatively higher MELD scores than the US will receive the organ. There is still insufficient effort to find the Korean MELD allocation system's current problems and solve these problems in such situation.

Thus, the aim of this study was to investigate changes in the distribution and outcomes of deceased donor liver transplantation before and after implementation of the MELD system. We analyzed the trend and raised a question as to whether there was any improvement for fair long-term distribution.

\section{MATERIALS AND METHODS}

We selected patients who underwent deceased donor liver transplantation at Seoul National University Hospital from June 2014 to June 2016. A total of 77 patients who received deceased donor liver transplantation based on the CTP score allocation system and 52 patients who were selected by the MELD allocation system from June 2016 to June 2018 were participants of this study. For comparison before and after the application of the MELD System, we reviewed clinical data of both groups, including sex, age, blood type, primary disease, MELD score, CTP score at the time of assignment, 6-month survival rate, post-transplant hospital stay, re-admission rate within three months, and complication rate within three months.

For statistical verification, statistical analysis was performed and continuous variables were compared by independent t-test using IBM SPSS version 23 (IBM Corp., Armonk, NY, USA). Chi-square test or Fisher exact test was used to compare cat-

Table 1. Preoperative findings of CTP and MELD allocation groups

\begin{tabular}{lccc}
\multicolumn{1}{c}{ Recipients variable } & $\begin{array}{c}\text { CTP group } \\
(\mathrm{n}=77)\end{array}$ & $\begin{array}{c}\text { MELD group } \\
(\mathrm{n}=52)\end{array}$ & p-value \\
\hline Age (yr) & $56.5 \pm 10.9$ & $53.4 \pm 1.16$ & 0.141 \\
Sex (male : female) & $49: 28$ & $36: 16$ & 0.511 \\
Blood type & & & 0.527 \\
A+ & $30(38.9)$ & $20(38.5)$ & \\
B+ & $18(23.4)$ & $11(21.2)$ & \\
O+ & $18(23.4)$ & $17(32.7)$ & \\
AB+ & $11(14.3)$ & $4(7.69)$ & \\
Diagnosis & & & \\
HBV LC & $32(41.6)$ & $22(42.3)$ & \\
HCV LC & $15(19.5)$ & $3(5.8)$ & \\
Alcoholic LC & $14(18.2)$ & $24(46.2)$ & \\
NBNC LC & $4(5.2)$ & $2(3.8)$ & \\
Biliary cirrhosis & $3(3.9)$ & 0.0001 & \\
Wilson's disease & $3(3.9)$ & $3(5.8)$ & \\
Fulminant hepatic failure & $3(3.9)$ & $0(0.0)$ & \\
Toxic hepatitis & $1(1.3)$ & $0(0.0)$ & \\
Primary non-function & $2(2.6)$ & $0(0.0)$ & \\
MELD score & $31.0 \pm 8.2$ & $37.8 \pm 2.0$ & \\
Child Pugh score (A/B/C) & $3 / 18 / 56$ & $1 / 0 / 51$ & \\
Hepatocellular carcinoma & $36(46.8)$ & $12(23.1)$ & \\
\hline
\end{tabular}

Values are presented as mean \pm standard deviation, number only, or number (\%).

CTP, Child-Turcotte-Pugh; MELD, Model for end-stage liver disease; HBV LC, hepatitis B-related liver cirrhosis; HCV LC, hepatitis C-related liver cirrhosis; NBNC LC, non-B non-C liver cirrhosis.
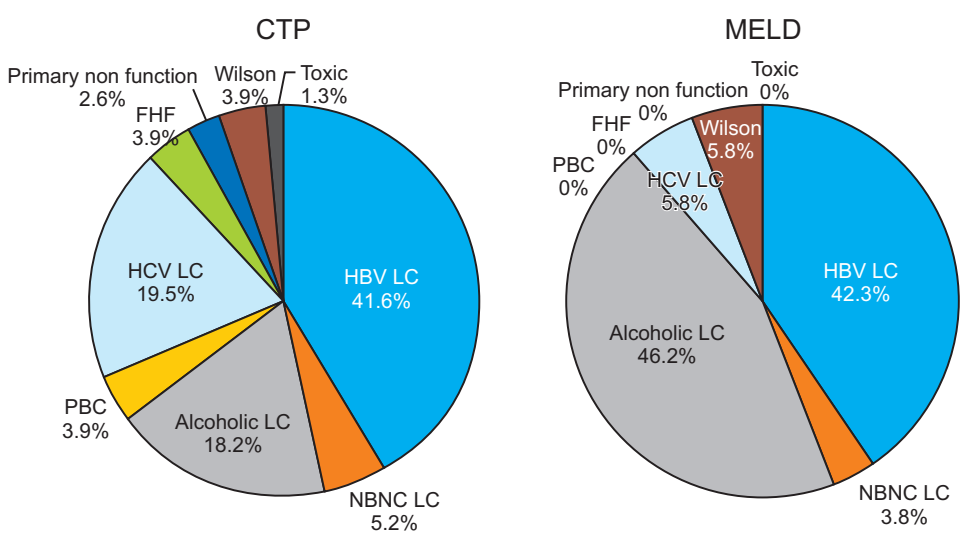

HBV LC

NBNC LC

Alcoholic LC

PBC

HCV LC

FHF

Primary

non function

Wilson

Toxic
Fig. 1. Changes of etiology between MELD and CTP based allocation systems. MELD, Model for End-Stage Liver Disease; CTP, Child-Turcotte-Pugh; LC, liver cirrhosis; HBV LC, hepatitis B-related liver cirrhosis; HCV LC, hepatitis C-related liver cirrhosis; PBC, primary biliary cirrhosis; FHF, fulminant hepatic failure; NBNC LC, non-B non-C liver cirrhosis. 
egorical variables. Survival rate was verified using the Cox proportional hazard model. A case where the $\mathrm{p}$-value was less than 0.05 was defined as statistically significant. This study was approved by the Institutional Review Board (IRB) of Seoul National University Hospital (SNUH) (IRB no. 2102-061-1195). Informed consent was waived by the IRB due to its retrospective study design. All procedures of this study were performed following the relevant guidelines and regulations.

\section{RESULTS}

\section{Preoperative findings and changes of etiology between MELD score and CTP based allocation systems}

A total of 129 patients who underwent deceased donor liver transplantation were included. Table 1 shows baseline characteristics and perioperative findings of subjects in MELD and CTP groups. There was no significant difference in age or sex between the two groups. Hepatitis B-related liver cirrhosis was the most common indication of the liver transplantation in both groups $(42.3 \%$ in the MELD group and $41.6 \%$ in the CTP group). Hepatitis $\mathrm{C}$ related liver cirrhosis was decreased in the MELD period (5.8\% in the MELD group and $19.5 \%$ in the CTP group). The proportion of alcoholic liver cirrhosis in the MELD period was significantly increased (Fig. 1). The mean MELD score of the MELD group was $37.8 \pm 2.0$, which was higher than that $(31.0 \pm 8.2)$ of the CTP group. The distribution of Child-Pugh scores in CTP periods was 3 for A, 18 for B, and 56 for $\mathrm{C}$. In the MELD period, the distribution of Child-Pugh score was 1 for $\mathrm{A}, 51$ for $\mathrm{C}$. The ratio for Child $\mathrm{C}$ was increased.

\section{Comparison of postoperative outcomes between CTP and MELD allocation groups}

Table 2 shows postoperative outcomes of both CTP and MELD allocation groups. The 90 -day complication rate (> Clavien-Dindo Grade III) was significantly higher in the MELD group than in the CTP group $(11.5 \%$ vs. $2.6 \%$; $p=0.040)$. The 6 -month mortality rate of the MELD group was $25.0 \%$, which was higher than that $(11.7 \%)$ in the CTP group $(p=0.022)$. There was no significant difference in the overall complication rate, 90-day re-admission rate, or intensive care unit/hospital stay between the CTP group and the MELD group. After reviewing causes of death at six months, sepsis, bleeding, and neurologic deficit were the most common ones in order. Sepsis accounted for the most proportion in the MELD group and the CTP group (4/13, 30.8\% in the MELD group vs. $3 / 9,33.3 \%$ in the CTP group). GVHD occurred in two cases only in the CTP group. Death from fatal bleeding after transplantation was relatively higher in the MELD group $(5 / 13,38.5 \%$ in the MELD group vs.1/9, 11.1\% in the CTP group).

Logistic regression analysis was performed to identify which factor affected the 6-month mortality after transplantation (Table 3). Logistic regression analysis showed that the MELD

Table 2. Comparison of postoperative outcomes between CTP and MELD allocation groups

\begin{tabular}{lccc}
\hline \multicolumn{1}{c}{ Postoperative outcome } & $\begin{array}{c}\text { CTP group } \\
(\mathrm{n}=77)\end{array}$ & $\begin{array}{c}\text { MELD group } \\
(\mathrm{n}=52)\end{array}$ & $p$-value \\
\hline $\begin{array}{l}\text { Overall 90 days } \\
\text { complication rate }\end{array}$ & $26(33.8)$ & $24(46.2)$ & 0.198 \\
$\begin{array}{l}\text { 90-day complication rate } \\
\text { (> Clavien-Dindo Grade III) }\end{array}$ & $2(2.6)$ & $6(11.5)$ & 0.040 \\
90-day re-admission & $46(59.7)$ & $34(65.4)$ & 0.517 \\
ICU stay (day) & $8.00 \pm 6.75$ & $9.76 \pm 11.3$ & 0.315 \\
Hospital stays (day) & $42.1 \pm 54.6$ & $32.2 \pm 35.6$ & 0.216 \\
6-month mortality & $9(11.7)$ & $13(25.0)$ & 0.022 \\
Cause of death & & & 0.356 \\
$\quad$ Sepsis & $3(33.3)$ & $4(30.8)$ & \\
Bleeding & $1(11.1)$ & $5(38.5)$ & \\
Neurologic deficit & $1(11.1)$ & $2(15.4)$ & \\
HCV reactivation & $1(11.1)$ & $0(0.0)$ & \\
HCC recurrence & $1(11.1)$ & $1(7.7)$ & \\
GVHD & $2(22.2)$ & $0(0.0)$ & \\
Primary non-function & $0(0.0)$ & $1(7.7)$ & \\
\hline
\end{tabular}

Values are presented as number (\%) or mean \pm standard deviation. CTP, Child-Turcotte-Pugh; MELD, Model for End-stage Liver Disease; ICU, intensive care unit; $\mathrm{HCV}$, hepatitis $\mathrm{C}$ virus; $\mathrm{HCC}$, hepatocellular carcinoma; GVHD, graft-versus-host disease.

Table 3. Risk factor analysis for 6-month mortality after liver transplantation

\begin{tabular}{|c|c|c|c|c|}
\hline \multirow{2}{*}{ Variable } & \multicolumn{2}{|c|}{ Univariate analysis } & \multicolumn{2}{|c|}{ Multivariate analysis } \\
\hline & $\mathrm{HR}(95 \% \mathrm{Cl})$ & $p$-value & $\mathrm{HR}(95 \% \mathrm{Cl})$ & $p$-value \\
\hline Age $(y r)$ & $1.040(0.998-1.084)$ & 0.060 & $1.040(0.993-1.090)$ & 0.095 \\
\hline Sex (male-female) & $1.095(0.410-2.926)$ & 0.857 & - & - \\
\hline ABO blood type & $2.106(0.706-6.281)$ & 0.269 & - & - \\
\hline MELD score & $1.005(0.943-1.071)$ & 0.874 & - & - \\
\hline Child Pugh score & $1.320(0.132-13.21)$ & 0.931 & - & - \\
\hline Hepatocellular carcinoma & $0.513(0.184-1.428)$ & 0.201 & - & - \\
\hline MELD/CTP allocation & $2.225(0.951-5.207)$ & 0.058 & $2.865(1.057-7.764)$ & 0.039 \\
\hline
\end{tabular}

HR, hazard ratio; Cl, confidence interval; MELD, Model for End-stage Liver Disease; CTP, Child-Turcotte-Pugh. 


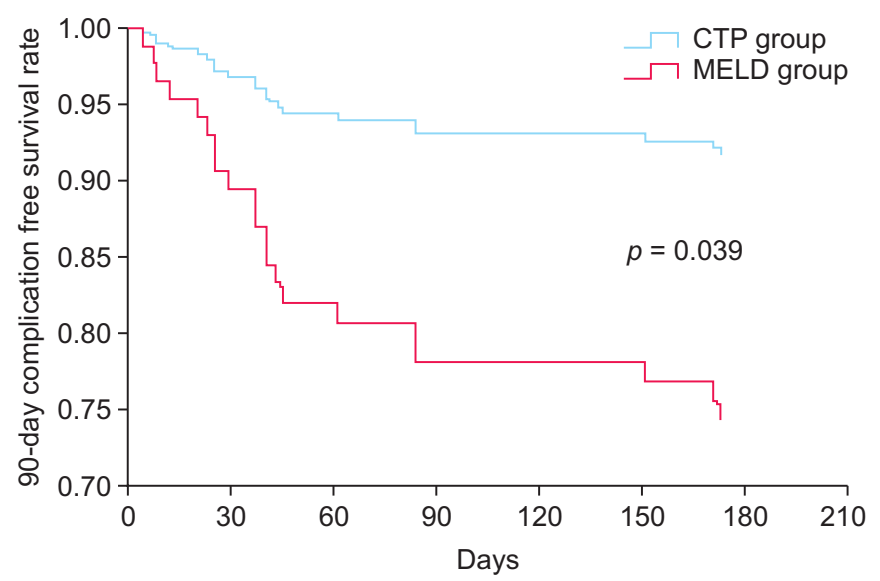

Fig. 2. Overall 6-month cumulative survival difference between MELD and CTP based allocation groups. MELD, Model for End-Stage Liver Disease; CTP, Child-Turcotte-Pugh.

eallocation system was associated with significantly higher 6-month survival rate and 90-day complication rate. In univariate analysis, age of recipients (hazard ratio [HR], 1.040; 95\% confidence interval [CI], 0.998-1.084; $p=0.060)$ and MELD allocation system (HR, 2.225; 95\% CI, 0.951-5.207, $p=0.058$ ) showed possible associations with 6-month mortality. In multivariate analysis, the MELD allocation system was the only factor that showed significant association with higher 6-month mortality than the CTP allocation system (HR, 2.865; 95\% CI, 1.057-7.764, $p=0.039$ ). Fig. 2 shows an overall 6-month cumulative survival curve between CTP and MELD allocation groups. The CTP group showed significantly $(p=0.039)$ more prolonged survival than the MELD allocation group. Fig. 3 shows 90-day complication-free survival curve of CTP and MELD allocation groups.

\section{DISCUSSION}

In the area of liver transplantation, the ultimate purpose of liver allocation is to allocate the liver to the person who needs it the most so that the person can live a healthy life. An index for evaluating this is the waiting list mortality of people who have registered with a responsible institution such as UNOS or KONOS for liver transplantation. The reason for the change from the CTP system to the MELD system was to reduce the waiting list mortality. This is because a patient's MELD score better reflects the waiting mortality rate than the CTP score. The MELD system succeeded in reducing the waiting list mortality in several countries $[2,3,11]$. Still, it is a controversial conclusion whether it can improve the long-term survival rate of liver transplant recipients.

Reports about the MELD allocation system have concluded that increasing complication rate and hospital stay are inevitable because patients in poor conditions receive liver trans-

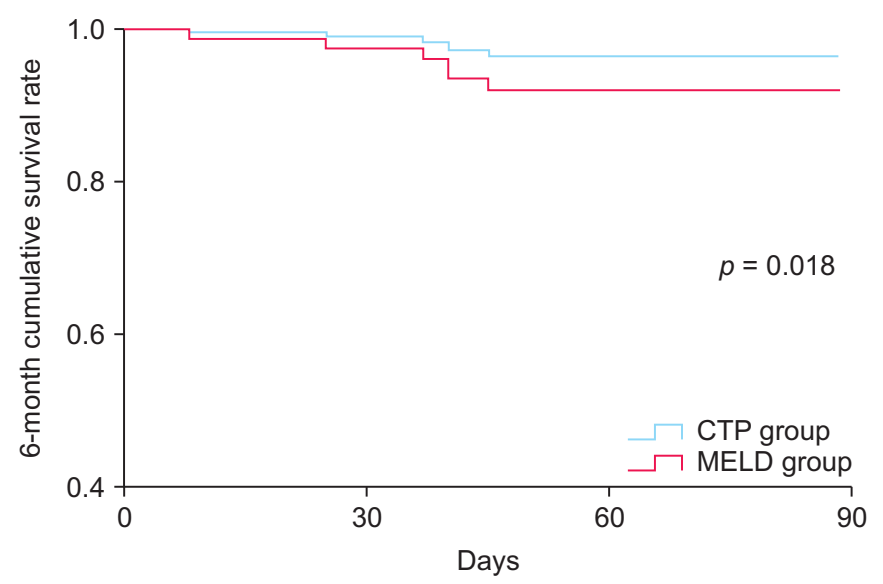

Fig. 3. Overall 90-day complication free survival difference between MELD and CTP based allocation groups. MELD, Model for End-Stage Liver Disease; CTP, Child-Turcotte-Pugh.

plantation. Despite that, there was no significant difference in survival rate such as the 1 -year survival rate $[2,7]$. However, patients with too poor conditions often die in immediate postoperative periods due to preoperative factors such as hidden infection, the progress of end-organ damage, and neurologic deficit even after a liver transplantation. In this respect, comparing the mortality rate at 30-day or 90-day after transplantation rather than one-year after transplantation could be more appropriate to discriminate allocation failure.

In this study, the 90-day complication rate and the 6-month mortality rate were higher in the MELD era than in the CTP allocation period. As for causes of death, sepsis-related bleeding, underlying coagulopathy, renal function decline, cardiac complication, neurological deficit, and lung function deterioration were not recovered even after liver transplantation.

Among causes of death, fatal postoperative bleeding was relatively higher in the MELD group. It occurred in recipients with size-mismatch of graft-to-recipients. The outflow compression by huge liver made the graft liver become enlarged, leading to fatal bleeding and graft failure.

The criteria for a successful organ distribution of deceased liver transplantation should consider the expected graft and patient survival with a reduction of waiting list mortality. No accurate tool for predicting mortality has been developed. However, it is necessary to allocate organs to consider the graft and the patient's survival rate by analyzing causes of those who died after liver transplantation, excluding marginal liver and technical problems.

Results of this study were similar to those of previous article on the use of the existing Korean MELD system. However, this article aims to provide a different perspective on the interpretation of the phenomenon in which complications and mortality are higher in the MELD group in terms of early outcome, not one-year performance, but a shorter early outcome. 
First, since the current system has been developed to reduce only waiting list mortality, a revision must be considered. In other words, it is necessary to prioritize and perform organ allocation in consideration of "expected Graft/patient survival."

Unfortunately, in this paper, we did not propose a new model or talk about alternatives. However, it has a tremendous significance in raising issues mentioned in existing papers, thus increasing our awareness about the problem of the current system. It can act as a catalyst for discussion and consensus on this problem.

Now, various transplant centers, including our center, are conducting studies on predictive models for mortality after liver transplantation. In the past, it was challenging to consider expected graft/patient survival because it was difficult to predict patient survival. However, with the introduction of new technologies such as machine learning/artificial intelligence, an environment has been created in which a model with a sufficiently high prediction rate can be built since a large amount of data are available now.

Several studies are now ongoing to predict survival rates relatively accurately with the development of new predictive models such as machine learning and artificial intelligence. It is necessary to change the system that allocates organs to consider both MELD score and the expected survival rate through various variables $[1,12]$.

However, since there is still no integrated national database for liver transplantation, it is necessary to conduct a transplant society project. The Ministry of Health and Welfare can improve this. If a large-scale DB and an optimization model can be implemented in the near future, a new allocation system that can optimize waiting list mortality and maximize expected graft/patient survival can be built by considering various variables.

However, ethical issues still remain. This is a limitation of this study. An organ allocation program should be revised considering expected graft survival for patients with high MELD scores. In that case, some might not receive an allocation and some may argue that this does not fit the defining principle of distribution. In addition, the expected graft survival might be inaccurate. In that case, it is necessary to consider those who are excluded from allocation and those who are likely to live after receiving a transplant.

However, if this system is maintained, $25 \%$ of livers will continue to be wasted on people who have a high chance of death. Thus, unnecessary medical resources will continue to be consumed during hospitalization. If we leave this without improving, we need to think about whether it fits the principle of justice.

In addition, we can see that the change in this system is decreasing the benefit rate for hepatitis B and hepatocellular carcinoma patients and increasing alcoholic liver disease patients. In Korea, a hepatitis B epidemic area, the hepatitis B vaccination project and the surveillance system are well estab- lished at the national level. Therefore, most hepatitis B patients receive regular follow ups in hospitals. With the development of potent antiviral agents such as entecavir and tenofovir, they can maintain a healthy life, not much different from the general population. Therefore, even if liver cirrhosis progresses, it progresses slowly and the patient's compliance is acceptable. Generous compliance of these patients is also reflected in their compliance with regular immunosuppressant use after liver transplantation. This might be one key factor contributing to the high long-term success rate of liver transplantation in Korea.

Family support is essential for alcoholic patients to abstain from alcohol and increase their compliance with immunosuppressants. However, most alcoholic patients have lousy family relationships with poor adherence $[13,14]$. Therefore, the rate of re-drinking is high. Acute cellular rejection occurs also due to their poor intake of immunosuppressants. Because of these mentioned factors, the long-term performance of a deceased donor liver transplant may decrease.

We have already achieved a dramatic reduction in waiting list mortality through system improvements. We have succeeded in allocating livers to the sickest patients. However, our ultimate goal is not only to distribute livers to them, but also to prolong their life through liver transplantation. We have been performing organ allocation with a MELD system for the past five years. It is now necessary to analyze results based on national data and create a newly refined allocation model. This will reduce waiting list mortality and increase the survival rate after liver transplantation. Subsequent research on this and feedback on results of the continuous allocation model should be done. There is no royal road to the liver transplant distribution model. Only constant feedback and improvement can increase patient survival.

\section{CONFLICT OF INTEREST}

No potential conflict of interest relevant to this article was reported.

\section{ORCID}

Han Sang Park, https://orcid.org/0000-0002-0006-2995 Jeong-Moo Lee, https://orcid.org/0000-0001-7806-8759 Kwangpyo Hong, https://orcid.org/0000-0001-7909-8116 Eui Soo Han, https://orcid.org/0000-0001-9582-1255 Suk Kyun Hong, https://orcid.org/0000-0002-0020-6215 YoungRok Choi, https://orcid.org/0000-0003-2408-7086 Nam-Joon Yi, https://orcid.org/0000-0002-5467-425X Kwang-Woong Lee, https://orcid.org/0000-0001-6412-1926 Kyung-Suk Suh, https://orcid.org/0000-0002-9535-7349 


\section{AUTHOR CONTRIBUTIONS}

Conceptualization: JML. Data curation: HSP. Methodology: JML, SKH. Visualization: KH, ESH. Writing - original draft: HSP, JML. Writing - review \& editing: SKH, YRC, NJY, KWL, KSS.

\section{REFERENCES}

1. Bertsimas D, Kung J, Trichakis N, Wang Y, Hirose R, Vagefi PA. Development and validation of an optimized prediction of mortality for candidates awaiting liver transplantation. Am J Transplant 2019;19:1109-1118.

2. Lee J, Lee JG, Jung I, Joo DJ, Kim SI, Kim MS. Development of a Korean liver allocation system using model for end stage liver disease scores: a nationwide, multicenter study. Sci Rep 2019;9:7495.

3. Wiesner R, Edwards E, Freeman R, Harper A, Kim R, Kamath P, et al. Model for end-stage liver disease (MELD) and allocation of donor livers. Gastroenterology 2003;124:91-96.

4. Thompson MA, Redden DT, Glueckert L, Smith AB, Crawford JH, Jones KA, et al. Risk factors associated with reoperation for bleeding following liver transplantation. HPB Surg 2014;2014:816246.

5. Massicotte L, Carrier FM, Karakiewicz P, Hevesi Z, Thibeault L, Nozza A, et al. Impact of MELD score-based organ allocation on mortality, bleeding, and transfusion in liver transplantation: a before-and-after observational cohort study. J Cardiothorac Vasc Anesth 2019;33:2719-2725.

6. Kamath PS, Kim WR. The model for end-stage liver disease (MELD).
Hepatology 2007;45:797-805.

7. Elwir S, Lake J. Current status of liver allocation in the United States. Gastroenterol Hepatol (N Y) 2016;12:166-170.

8. Wedd JP, Harper AM, Biggins SW. MELD score, allocation, and distribution in the United States. Clin Liver Dis (Hoboken) 2013;2:148151.

9. Lee JA, Choi GS, Kim JM, Kwon CH, Joh JW. Comparison study of outcomes of deceased donor liver transplantation before and after Korean model for end-stage liver disease (MELD) system: single center experience. J Korean Soc Transplant 2018;32:7-11.

10. Croome KP, Lee DD, Burns JM, Keaveny AP, Taner CB. Intraregional model for end-stage liver disease score variation in liver transplantation: disparity in our own backyard. Liver Transpl 2018;24:488-496.

11. Jurado-García J, Muñoz García-Borruel M, Rodríguez-Perálvarez ML, Ruíz-Cuesta P, Poyato-González A, Barrera-Baena P, et al. Impact of MELD allocation system on waiting list and early post-liver transplant mortality. PLoS One 2016;11:e0155822.

12. Lau L, Kankanige Y, Rubinstein B, Jones R, Christophi C, Muralidharan $\mathrm{V}$, et al. Machine-learning algorithms predict graft failure after liver transplantation. Transplantation 2017;101:e125-e132.

13. Marroni CA, Fleck AM Jr, Fernandes SA, Galant LH, Mucenic M, de Mattos Meine $\mathrm{MH}$, et al. Liver transplantation and alcoholic liver disease: history, controversies, and considerations. World J Gastroenterol 2018;24:2785-2805.

14. López-Navas A, Ríos A, Riquelme A, Martínez-Alarcón L, Pons JA, Miras M, et al. Psychological care: social and family support for patients awaiting a liver transplant. Transplant Proc 2011;43:701-704. 